\title{
Early postoperative bleeding is independently associated with increased surgical mortality in infants after cardiopulmonary bypass
}

\author{
Michael J. Wolf, MD, ${ }^{a}$ Kevin O. Maher, MD, ${ }^{a}$ Kirk R. Kanter, MD, ${ }^{b}$ Brian E. Kogon, MD, ${ }^{b}$ \\ Nina A. Guzzetta, MD, ${ }^{\mathrm{c}}$ and William T. Mahle, $\mathrm{MD}^{\mathrm{a}}$
}

Objective: Infants undergoing cardiac surgery often have postoperative bleeding contributing to the occurrence of adverse events. A quantitative evaluation of postoperative bleeding has not been well described.

Methods: We identified 1071 infants who had undergone cardiopulmonary bypass from August 1, 2008 to December 31, 2011. The volume of postoperative bleeding and its effect on mortality were reviewed.

\begin{abstract}
Results: Postoperative bleeding during the first 12 hours postoperatively was stratified by quartiles. Bleeding was significantly associated with increased mortality (odds ratio [OR], 1.15; 95\% confidence interval [CI] $1.10-1.21 ; P<.001)$. Other risk factors significantly associated with mortality included greater Risk Adjustment for Congenital Heart Surgery score (OR, $1.5 ; 95 \%$ CI, 1.22-1.85; $P<.001)$, single ventricle anatomy (OR, 3.09; $95 \% \mathrm{CI}, 1.68-5.67 ; P<.001)$, younger age (OR, $0.99 ; 95 \% \mathrm{CI}, 0.98-0.99 ; P<.001)$, and longer perfusion time $(\mathrm{OR}, 1.01 ; 95 \% \mathrm{CI}, 1.01-1.02 ; P<.001)$. Subjects with greater bleeding volumes experienced a longer postoperative mechanical ventilation and intensive care unit stay. The overall hospital mortality was $4.1 \%$. On multivariate analysis, adjusting for age, single ventricle anatomy, Risk Adjustment for Congenital Heart Surgery score, and perfusion time, an increasing bleeding volume was independently associated with increased mortality. Packed red blood cell transfusion was independently associated with an increased duration of mechanical ventilation $(P=.01)$ and intensive care unit length of stay $(P=.003)$.

Conclusions: Early postoperative hemorrhage was independently associated with an increased mortality in infants after cardiac surgery. The longer interval from surgery to death suggests that other factors, aside from the bleeding itself, including the transfusion volume, might contribute to mortality. Initiatives to limit postoperative bleeding and to critically appraise packed red blood cell transfusion practices are warranted. (J Thorac Cardiovasc Surg 2014;148:631-6)
\end{abstract}

Supplemental material is available online.

Infants who undergo cardiac surgery within the first year of life often experience significant postoperative bleeding after admission to the intensive care unit (ICU). ${ }^{1}$ In addition to the extensive suture lines, increased hemodilution, activation of the coagulation system, and hemostatic derangements after cardiopulmonary bypass (CPB) increase the risk of postoperative bleeding. ${ }^{1-4}$ Inherent deficiencies in antithrombin and other coagulation proteins can also prevent adequate hemostasis during and after $\mathrm{CPB} .{ }^{5,6}$

\footnotetext{
From the Division of Pediatric Cardiology, ${ }^{\mathrm{a}}$ Department of Pediatrics, Division of Pediatric Cardiothoracic Surgery, ${ }^{\mathrm{b}}$ Department of Surgery, and Division of Pediatric Cardiac Anesthesia, ${ }^{c}$ Department of Anesthesia, Emory University School of Medicine, Atlanta, Ga.

Disclosures: Authors have nothing to disclose with regard to commercial support.

Received for publication Feb 20, 2013; revisions received Sept 12, 2013; accepted for publication Oct 29, 2013; available ahead of print Dec 10, 2013.

Address for reprints: Michael J. Wolf, MD, Division of Pediatric Cardiology, Department of Pediatrics, Emory University School of Medicine Children's Healthcare of Atlanta, 1405 Clifton Rd NE, Atlanta, GA 30322 (E-mail: wolfm@kidsheart.com). $0022-5223 / \$ 36.00$

Copyright (C) 2014 by The American Association for Thoracic Surgery http://dx.doi.org/10.1016/j.jtcvs.2013.10.050
}

Excessive postoperative bleeding will often be treated with blood products, including packed red blood cells (PRBCs), fresh frozen plasma, platelets, and thawed cryoprecipitate. In cases of severe bleeding, activated factor VII will often be administered to achieve adequate hemostasis. ${ }^{7}$ Laboratory tests, including the thromboelastogram, have allowed for specific targeting of deficient areas of the coagulation system during ongoing bleeding. ${ }^{8}$ When factor and product replacement have failed to achieve hemostasis, surgical exploration will usually be undertaken.

Excessive postoperative bleeding requires replacement with blood transfusions. Although safe during administration, blood transfusions carry several risks that can affect morbidity and mortality after cardiac surgery. Adult data have demonstrated increased mortality risk in association with PRBC transfusion after coronary artery bypass grafting ${ }^{9}$ and a significant effect on long- and short-term survival after all types of adult heart surgery. ${ }^{10}$ Pediatric data have demonstrated an increased duration of mechanical ventilation and ICU length of stay in relation to blood transfusions, with no demonstrable survival benefit for aggressive transfusion strategies compared with conservative transfusion strategies. ${ }^{11-17}$

The primary goal of the present study was to examine the relationship of early postoperative bleeding in infants with 


\section{Abbreviations and Acronyms \\ $\mathrm{CPB}=$ cardiopulmonary bypass \\ ICU = intensive care unit \\ PRBC $=$ packed red blood cell \\ RACHS $=$ Risk Adjustment for Congenital Heart Surgery}

surgical mortality and adverse events. We hypothesized that increasing amounts of postoperative bleeding would be associated with an increased mortality risk and adverse events, including a prolonged duration of mechanical ventilation and ICU length of stay. In particular, we focused on a quantitative evaluation of early postoperative bleeding after infant cardiac surgery and its effect on mortality.

\section{METHODS}

\section{Study Design}

The Children's Healthcare of Atlanta institutional review board approved the present study. Data were collected from the individual medical records and our institutional surgical database. All infants $<1$ year of age who had undergone cardiac surgery with CPB from August 1, 2008 through December 31, 2011 were included in the present analysis. Early postoperative bleeding was defined as bleeding that occurred within the first 12 hours after ICU admission. All patients included in the analysis were treated in the cardiac ICU postoperatively.

In addition to the volume of postoperative bleeding, the data collected included age, weight, height, cardiac anatomy, presence of chromosomal anomaly, Risk Adjustment for Congenital Heart Surgery (RACHS) score, perfusion time in the operating room, blood products received in the operating room, blood products received in the ICU, mechanical ventilation duration, length of ICU stay, in-hospital mortality, postoperative cardiac arrest, postoperative infection confirmed by positive wound culture or blood culture findings, delayed sternal closure, need for surgical re-exploration, need for mechanical circulatory support, and need for continuous renal replacement therapy.

Each operative admission was treated separately. In patients who underwent $>1$ operation during an admission, the primary operation for which they had been admitted to the hospital was chosen for analysis. As such, the patients who underwent a stage I Norwood operation as a newborn and a subsequent cavopulmonary anastomosis at 4 months of age were included in the analysis as 2 unique encounters.

The CPB anticoagulation protocol at the Children's Healthcare of Atlanta during the entire study period consisted of a single bolus dose of $500 \mathrm{U} / \mathrm{kg}$ of unfractionated heparin immediately before the initiation of CPB. Monitoring was achieved with activated clotting times, with a goal of $>500$ seconds. Repeat doses of heparin $(100 \mathrm{U} / \mathrm{kg})$ were given to maintain the activated clotting time $>500$ seconds throughout the duration of CPB. Aminocaproic acid (Amicar; Clover Pharmaceuticals, Marietta, $\mathrm{Ga}$ ) was routinely administered to infants undergoing repeat sternotomy. After CPB, a dose of protamine $(5 \mathrm{mg} / \mathrm{kg})$ was administered to reverse anticoagulation. Ongoing bleeding in the operating room was treated with platelets, thawed cryoprecipitate, and fresh frozen plasma. In cases of severe bleeding for which a surgical cause could not be identified, activated factor VII was used.

The vast majority of patients underwent bicaval cannulation and moderate hypothermia $\left(28^{\circ}-32^{\circ} \mathrm{C}\right)$, except for neonates undergoing aortic arch reconstruction or a first stage Norwood procedure, for whom hypothermia to $18^{\circ} \mathrm{C}$ was typically used with regional cerebral perfusion. The use of profound hypothermic circulatory arrest for $>2$ or 3 minutes was rare. The hematocrit was maintained at $>28 \%$, often with the use of hemofiltration during CPB. Modified venovenous ultrafiltration at the termination of $\mathrm{CPB}$, particularly in neonates was commonly (but not universally) used, according to surgeon preference. The cell saver was also available for virtually all patients and was generally preferred over PRBCs both in the operating room and in the ICU.

Patients who continued to have significant bleeding on admission to the cardiac ICU were treated further with multiple blood products, including fresh frozen plasma, platelets, thawed cryoprecipitate, and PRBCs. In severe cases, repeat dosing of activated factor VII was used. The coagulation tests used included the prothrombin time, activated partial thromboplastin time, international normalized ratio, and anti-Xa assay. Thromboelastography and platelet mapping were also used more recently to help target specific areas of the coagulation system, if the bleeding was difficult to control. When the bleeding could not be controlled medically, or if signs or symptoms of pericardial tamponade developed, surgical exploration was undertaken to identify a specific source of bleeding.

The primary outcome of the present study was in-hospital mortality. Secondary outcomes were the mechanical ventilation duration and length of ICU stay.

\section{Statistical Analysis}

Statistical analysis was performed using STATA, version 12.0, software (StataCorp LP, College Station, Tex) and Statistical Analysis Software, version 9.3 (SAS Institute Inc, Cary, NC). Univariate analysis was performed using Student's $t$ test or analysis of variance for continuous data and Fisher's exact test or $\chi^{2}$ for categorical data. Multivariate logistic and linear regression analysis was undertaken to adjust for covariates. To predict the effect of postoperative bleeding on mortality risk, all variables with $P<.1$ on univariate analysis were entered into a multivariate logistic regression analysis.

\section{RESULTS}

A total of 1071 infants $<1$ year of age underwent cardiac surgery with CPB during the study period. To quantify the bleeding amounts, the patients were stratified by quartile according to the quantity of bleeding during the first 12 postoperative hours: 0 to $1.19,1.20$ to $2.00,2.01$ to 4.19 , and $\geq 4.20 \mathrm{~mL} / \mathrm{kg} / \mathrm{h}$. The patient characteristics are summarized in Table 1.

The patients in the higher quartiles were younger, weighed less, and had greater RACHS scores and longer perfusion times $(P<.001)$. Patients in the highest quartile had a median age of 8 days (range, 0-346) compared with a median age of 103 to 158 days in the 3 lower quartiles $(P<.001)$. The median weight in the highest quartile was $3.4 \mathrm{~kg}$ (range, 2.0-8.7) compared with a median weight of 4.4 to $5.8 \mathrm{~kg}$ in the 3 lower quartiles $(P<.001)$. The median RACHS score in the 2 highest quartiles was 3 compared with a score of 2 in the 2 lower quartiles $(P<.001)$. The perfusion time also increased with increasing quartile. The median perfusion time in the lowest quartile was 76 minutes (range, 18-211), increasing to 139 minutes in the highest quartile (range, $31-361 ; P<.001$ ).

Single ventricle anatomy was present in $22 \%$ to $30 \%$ of the patients across the quartiles, with a trend toward statistical significance $(P=.08)$. Chromosomal anomalies were present in $11 \%$ to $17 \%$, and heterotaxy syndrome ranged 
TABLE 1. Patient characteristics

\begin{tabular}{|c|c|c|c|c|c|}
\hline \multirow[b]{2}{*}{ Characteristic } & \multicolumn{4}{|c|}{ Bleeding quartile $(\mathrm{mL} / \mathrm{kg} / \mathrm{h})$} & \multirow[b]{2}{*}{$P$ valu } \\
\hline & 0-1.18 & 1.19-2.00 & 2.01-4.19 & $\geq 4.2$ & \\
\hline Patients (n) & 265 & 268 & 268 & 270 & - \\
\hline Age (d) & $152(1-363)$ & $138(1-356)$ & $103(1-336)$ & $8(0-346)$ & .001 \\
\hline Weight $(\mathrm{kg})$ & $5.8(2.4-10.3)$ & $5.5(2.0-10.8)$ & $4.4(2.0-9.8)$ & $3.4(2.0-8.7)$ & .001 \\
\hline RACHS & $2(1-6)$ & $2(1-6)$ & $3(2-6)$ & $3(1-6)$ & .001 \\
\hline Single ventricle & $74(28)$ & $59(22)$ & $60(22)$ & $81(30)$ & .08 \\
\hline Chromosomal anomaly & $46(17)$ & $30(11)$ & $34(13)$ & $42(16)$ & .17 \\
\hline Heterotaxy & $12(5)$ & $11(4.1)$ & $5(1.9)$ & $12(4)$ & .31 \\
\hline
\end{tabular}

Data presented as median (range) or $\mathrm{n}(\%)$. RACHS, Risk adjustment for congenital heart surgery.

from $1.9 \%$ to $5 \%$, with no significant difference across the quartiles. The surgical details and outcomes are summarized in Table 2.

Mortality increased across the quartiles, with $10 \%$ mortality in the highest bleeding quartile, $4.5 \%$ in the third, $1.1 \%$ in the second, and $0.4 \%$ in the lowest quartile $(P<.001)$. The overall hospital mortality for all patients analyzed was $4.1 \%$. The interval from surgery to death was similar in the second and fourth quartiles (median, 16 and 14 days, respectively) and longest in the third quartile (median, 21 days). One death occurred in the lowest quartile 1 day after surgery (Figure 1 ).

The PRBC volume transfused increased across the quartiles, with patients in the highest quartile receiving a median of $8.8 \mathrm{~mL} / \mathrm{kg}$ (range, 0-395) of transfused blood during the first 12 postoperative hours. The median PRBC volume transfused was 0 for the 3 lower quartiles, although the mean volume increased from $3.0 \mathrm{~mL} / \mathrm{kg}$ within 12 hours in the lowest quartile to $6.7 \mathrm{~mL} / \mathrm{kg}$ within 12 hours in the third quartile. Of the 1071 patients in our cohort, $644 \mathrm{did}$ not receive any PRBC transfusions after surgery. The cell saver that was administered was not considered transfused
PRBCs. The mean volume transfused in the highest quartile was $22 \mathrm{~mL} / \mathrm{kg}$ within 12 hours.

The duration of mechanical ventilation increased across the quartiles, with the lowest 3 quartiles having a median duration of 22 to 42 hours and the highest quartile, a median duration of 119 hours (range, $0-3632 ; P<.001$ ). The ICU length of stay was similar, with a median range of 51 to 93 hours in the 3 lowest quartiles and 180 hours (range, 22-2931) in the highest quartile $(P<.001$; Figure E1).

Other factors that increased across quartiles included mechanical circulatory support, postoperative cardiac arrest, delayed sternal closure, postoperative renal replacement therapy (continuous venovenous hemofiltration), and reoperation to control bleeding. The infection rates did not differ significantly across the quartiles.

Using multivariate logistic regression analysis, after adjustment for age, weight, single ventricle anatomy, RACHS score, and perfusion time, an increasing bleeding quartile was independently associated with mortality risk, with an odds ratio of 1.15 (95\% confidence interval 1.07 $1.22 ; P<.001)$. Although PRBC transfusion emerged as a significant risk factor for mortality on univariate analysis

TABLE 2. Surgical details and outcomes

\begin{tabular}{|c|c|c|c|c|c|}
\hline \multirow[b]{2}{*}{ Variable } & \multicolumn{4}{|c|}{ Bleeding quartile (mL/kg/h) } & \multirow[b]{2}{*}{$P$ value } \\
\hline & 0-1.18 & 1.19-2.00 & 2.01-4.19 & $\geq 4.2$ & \\
\hline Patients (n) & 265 & 268 & 268 & 270 & - \\
\hline Postoperative ECMO & $1(0.4)$ & $4(1.5)$ & $9(3.4)$ & $25(9.3)$ & .001 \\
\hline Postoperative cardiac arrest & $5(1.9)$ & $6(2.2)$ & $6(2.2)$ & $32(12)$ & .001 \\
\hline Postoperative infection & $6(2.3)$ & $5(1.9)$ & $13(4.9)$ & $16(5.9)$ & .03 \\
\hline Open sternum & $6(2.3)$ & $11(4.1)$ & $43(16)$ & $103(38)$ & .001 \\
\hline Postoperative CVVH & $1(0.4)$ & $0(0)$ & $3(1.1)$ & $14(5.2)$ & .001 \\
\hline Reoperation for bleeding & $2(0.8)$ & $3(1.1)$ & $1(0.4)$ & $16(5.9)$ & .001 \\
\hline Perfusion time (min) & $76(18-211)$ & $81(18-315)$ & $103(24-307)$ & $139(31-361)$ & .001 \\
\hline $\mathrm{PRBC}(\mathrm{mL} / \mathrm{kg} / 12 \mathrm{~h})$ & $0(0-48)$ & $0(0-41)$ & $0(0-48)$ & $8.8(0-395)$ & .001 \\
\hline Ventilator duration (h) & $22(0-2233)$ & $23(0-3247)$ & $42(0-3248)$ & $119(0-3632)$ & .001 \\
\hline ICU length of stay (h) & $51(12-2680)$ & $51(18-1597)$ & $93(22-2038)$ & $180(22-2931)$ & .001 \\
\hline Death & $1(0.4)$ & $3(1.1)$ & $12(4.5)$ & $27(10)$ & .001 \\
\hline Interval from surgery to death (d) & 1 & $16(7-113)$ & $21(3-100)$ & $14(0-201)$ & .001 \\
\hline
\end{tabular}




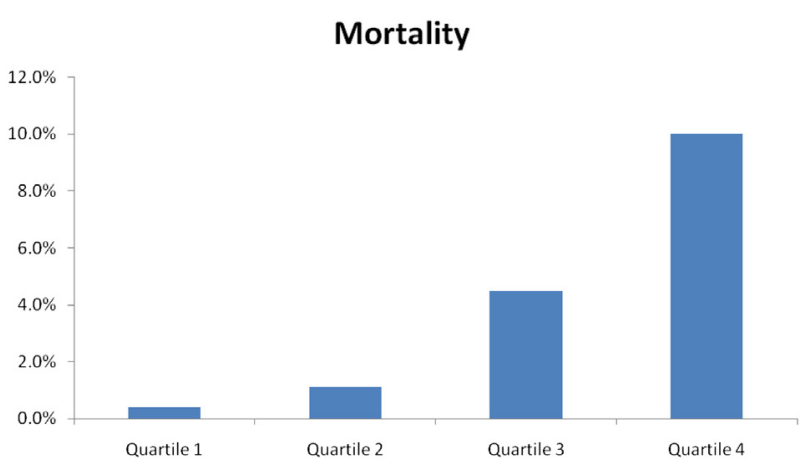

FIGURE 1. Hospital mortality rates stratified by quartile.

(odds ratio, 2.25; 95\% confidence interval, 1.22-4.16; $P<.001)$, it did not reach statistical significance in our multivariate model (odds ratio, $1.58 ; 95 \%$ confidence interval, $0.71-3.53 ; P=.3$ ). The univariate and multivariate analysis results are summarized in Tables 3 and 4 .

The effect of postoperative bleeding and PRBC transfusion on the duration of mechanical ventilation and ICU length of stay was also assessed using multivariate linear regression analysis. Controlling for age, RACHS score, perfusion time, and single ventricle anatomy, the effect of bleeding volume and PRBC transfusion was assessed. Patients with a greater bleeding volume had a longer duration of mechanical ventilation $(P<.001)$ and ICU length of stay $(P=.005)$. Patients who received PRBC transfusions also had a longer mechanical ventilation duration $(P=.01)$ and ICU length of stay $(P=.003)$.

\section{DISCUSSION}

This is the first study to our knowledge to quantitatively evaluate the effect of postoperative bleeding on hospital mortality risk after infant cardiac surgery. Although previous studies have examined the risk factors for increased postoperative blood loss, ${ }^{1,4}$ the effect on increased mortality risk was not described. In addition, the adverse effects of blood transfusions, including a longer duration of mechanical ventilation and ICU stay, have been well described in the pediatric data ${ }^{11,12,18}$; however, the effect on mortality risk has not been.

TABLE 3. Univariate mortality risk model

\begin{tabular}{llcc}
\hline \multicolumn{1}{c}{ Risk factor } & OR & $\mathbf{9 5} \%$ CI & $\boldsymbol{P}$ value \\
\hline Age & 0.99 & $0.98-0.99$ & $<.001$ \\
Single ventricle & 3.09 & $1.68-5.67$ & $<.001$ \\
RACHS & 1.5 & $1.22-1.85$ & $<.001$ \\
Perfusion time & 1.01 & $1.01-1.02$ & $<.001$ \\
Bleeding (mL/kg/h) & 1.15 & $1.10-1.21$ & $<.001$ \\
PRBCs (yes vs no) & 2.25 & $1.22-4.16$ & $<.001$ \\
PRBC (per 100 mL & 1.33 & $1.14-1.55$ & $<.001$ \\
$\quad$ transfused) & & & \\
\hline OR, Odds
\end{tabular}

$O R$, Odds ratio; $C I$, confidence interval; $R A C H S$, Risk Adjustment for Congenital Heart Surgery; $P R B C$, packed red blood cell.
TABLE 4. Multivariate mortality risk adjustment

\begin{tabular}{lccc}
\hline \multicolumn{1}{c}{ Risk factor } & OR & $\mathbf{9 5} \%$ CI & $\boldsymbol{P}$ value \\
\hline Age & 0.99 & $0.98-0.99$ & .002 \\
Single ventricle & 2.09 & $0.90-4.84$ & .0853 \\
RACHS & 1.06 & $0.80-1.41$ & .6901 \\
Perfusion time & 1.00 & $0.99-1.01$ & .4459 \\
Bleeding (mL/kg/h) & 1.15 & $1.07-1.22$ & $<.001$ \\
PRBC (yes vs no) & 1.58 & $0.71-3.53$ & .2669 \\
PRBC (per 100 mL & 0.99 & $0.73-1.32$ & .9187 \\
$\quad$ transfused) & & &
\end{tabular}

OR, Odds ratio; $C I$, confidence interval; $R A C H S$, Risk Adjustment for Congenital Heart Surgery; $P R B C$, packed red blood cell.

We identified several risk factors for increased blood loss in the postoperative period. In agreement with the findings of Williams and colleagues, ${ }^{1,4}$ younger age and lower weight both increased the risk and quantity of postoperative bleeding. A greater RACHS score and longer perfusion time were also associated with increased mortality risk and quantity of bleeding. The bleeding risk was not affected by the presence of a chromosomal anomaly, or heterotaxy syndrome, although single ventricle anatomy was associated with increased mortality risk on univariate analysis.

The mortality risk was significantly affected by an increased quantity of bleeding, with the risk of death more than doubling with each increased quartile. Aside from 1 patient in the lowest quartile who died on postoperative day 1 , the median interval from surgery to death ranged from 16 to 24 days in the 3 highest quartiles. Several factors could have influenced these intervals. Blood transfusions are known to cause inflammation, especially in the pulmonary system, when given in large quantities, known as transfusion-associated lung injury. ${ }^{19,20}$ Infants who experience significant postoperative hemorrhage tended to receive larger quantities of transfused blood products, increasing their risk of transfusion-associated lung injury. With increased lung inflammation, the duration of mechanical ventilation and its associated complications, including increased mortality, increased. Other factors that influenced the increased mortality risk were the greater incidence of postoperative cardiac arrest, mechanical circulatory support, and renal replacement therapy, all of which are independent risk factors for mortality after infant cardiac surgery.

The duration of mechanical ventilation and ICU length of stay were both independently affected by an increasing volume of postoperative bleeding. This remained statistically significant when controlling for other significant confounding variables. We attributed this to several factors. In addition to those mentioned, the patients with ongoing bleeding were more likely to return from the operating room with an open sternum and require subsequent surgical exploration because of bleeding, both of which increased the duration of mechanical ventilation and ICU length of stay. 
However, the infection rates did not differ across the quartiles. This was in contrast to the findings of several previous studies that identified delayed sternal closure, length of ICU stay, and duration of mechanical ventilation as risk factors for postoperative infection. ${ }^{21-23}$ All patients at our institution were treated with perioperative cefazolin for the first 48 hours after surgery, and those with an open sternum were treated with vancomycin and gentamycin until 24 hours after delayed sternal closure. These preemptive antibiotics might have lowered the infection risk across all groups. In addition, the objective of the present study was to analyze the association of postoperative bleeding with the clinical outcomes. The study was not designed to assess the effect of red blood cell transfusion per se on outcomes.

Blood transfusions are frequently given after infant cardiac surgery, and their adverse effect on outcomes has continued to emerge. Although the primary goal of the present study was to identify the increased mortality risk associated with increasing quantities of postoperative bleeding, PRBC transfusions were found to increase mortality risk. Although this effect did not remain on multivariate analysis, we raise the concern that the need for increased transfusions in the setting of significant bleeding plays a role in increasing mortality risk. Given the retrospective nature of the present study, it was difficult to differentiate the exact contributions that early postoperative bleeding and the resultant blood transfusions had on increased mortality risk.

We found an independent relationship between PRBC transfusion and an increased mechanical ventilation duration and ICU length of stay. These findings were in line with the findings of Salvin and colleagues ${ }^{11}$ and Kipps and colleagues. ${ }^{12}$

From our results, we stress the importance achieving adequate hemostasis in the operating room before ICU admission. All the risk factors identified in the present study occurred postoperatively in the ICU. By meticulously controlling postoperative bleeding in the operating room, a significant effect could be made on patient mortality. Although it is self-evident that 1 of the goals of congenital heart surgery is to minimize bleeding, strategies to achieve hemostasis must be balanced against the potential risks. Aggressive strategies to establish hemostasis could induce a prothrombotic state, leading thrombotic vessel occlusion. Some centers have advocated the use of fresh whole blood to minimize inflammation and induce a more favorable anticoagulation profile. Others have suggested that the use of aprotinin (currently not available in the United States) might lessen postoperative bleeding. ${ }^{14,24-26}$ In addition, given the independent association of blood transfusions and mortality, we stress the importance of weighing the risks and benefits of each transfused blood product, especially PRBCs, before administration. Their potential benefits, including increased oxygen carrying capacity and improved hemodynamics, have been well described ${ }^{16,18}$; however, the adverse effects are an important consideration, even for patients with ongoing bleeding. ${ }^{27-30}$

The present study had several important limitations. This was a single-center, retrospective analysis, and practices regarding hemostasis and the treatment of ongoing bleeding differ across institutions. In addition, several confounders were present that influenced the primary and secondary outcomes of the present study. Although these were controlled for in our multivariate analysis, their independent effect cannot be ignored. Although the independent association of an increasing quantity of postoperative bleeding and mortality was convincing, the association of transfusion was less so. A randomized controlled trial to analyze the independent effect of blood transfusions after infant cardiac surgery is necessary to provide definitive guidelines for their use.

\section{CONCLUSIONS}

Early postoperative hemorrhage after infant cardiac surgery was independently associated with increased inhospital mortality risk, a longer duration of mechanical ventilation, and an increased ICU length of stay. The longer interval from surgery to death seems to indicate that several mechanisms, other than bleeding itself and that might include factors such as inflammation and transfusion volume, could contribute to mortality. PRBC transfusions were also independently associated with an increased duration of mechanical ventilation and ICU length of stay. Initiatives to limit postoperative bleeding and critically appraise PRBC transfusion practices are warranted.

We acknowledge the work of Alexandra Ehrlich, MPH, on the statistical portion of our report.

\section{References}

1. Williams GD, Bratton SL, Riley EC, Ramamoorthy C. Association between age and blood loss in children undergoing open heart operations. Ann Thorac Surg. 1998;66:870-5; discussion 5-6.

2. Kern FH, Morana NJ, Sears JJ, Hickey PR. Coagulation defects in neonates during cardiopulmonary bypass. Ann Thorac Surg. 1992;54:541-6.

3. Chan AK, Leaker M, Burrows FA, Williams WG, Gruenwald CE, Whyte L, et al Coagulation and fibrinolytic profile of paediatric patients undergoing cardiopulmonary bypass. Thromb Haemost. 1997;77:270-7.

4. Williams GD, Bratton SL, Ramamoorthy C. Factors associated with blood loss and blood product transfusions: a multivariate analysis in children after openheart surgery. Anesth Analg. 1999;89:57-64.

5. Andrew M, Paes B, Milner R, et al. Development of the human coagulation system in the full-term infant. Blood. 1987;70:165-72.

6. Gruenwald CE, Manlhiot C, Crawford-Lean L, Foreman C, Brandao LR, McCrindle BW, et al. Management and monitoring of anticoagulation for children undergoing cardiopulmonary bypass in cardiac surgery. J Extra Corpor Technol. 2010;42:9-19.

7. Winch PD, Naguib AN, Bradshaw JR, Galantowicz M, Tobias JD. Decreasing the need for transfusion: infant cardiac surgery using hemodilution and recombinant factor VIIa. Pediatr Cardiol. 2013;34:119-24.

8. Weitzel NS, Weitzel LB, Epperson LE, Karimpour-Ford A, Tran ZV, Seres T. Platelet mapping as part of modified thromboelastography (TEG(R)) in patients 
undergoing cardiac surgery and cardiopulmonary bypass. Anaesthesia. 2012;67: 1158-65.

9. DeFoe GR, Ross CS, Olmstead EM, Surgenor SD, Fillinger MP, Groom RC, et al. Lowest hematocrit on bypass and adverse outcomes associated with coronary artery bypass grafting. Northern New England Cardiovascular Disease Study Group. Ann Thorac Surg. 2001;71:769-76.

10. Bhaskar B, Dulhunty J, Mullany DV, Fraser JF. Impact of blood product transfusion on short and long-term survival after cardiac surgery: more evidence. Ann Thorac Surg. 2012;94:460-7.

11. Salvin JW, Scheurer MA, Laussen PC, Wypij D, Polito A, Bacha EA, et al. Blood transfusion after pediatric cardiac surgery is associated with prolonged hospital stay. Ann Thorac Surg. 2011;91:204-10.

12. Kipps AK, Wypij D, Thiagarajan RR, Bacha EA, Newburger JW. Blood transfusion is associated with prolonged duration of mechanical ventilation in infants undergoing reparative cardiac surgery. Pediatr Crit Care Med. 2011;12: 52-6.

13. Blackwood J, Joffe AR, Robertson CM, Dinu IA, Alton G, Penner K, et al. Association of hemoglobin and transfusion with outcome after operations for hypoplastic left heart. Ann Thorac Surg. 2010;89:1378-84, e1-2.

14. Bojan M, Boulat C, Peperstraete H, Pouard P. High-dose aprotinin, blood product transfusions, and short-term outcome in neonates and infants: a pediatric cardiac surgery center experience. Paediatr Anaesth. 2012;22:818-25.

15. Cholette JM, Rubenstein JS, Alfieris GM, Powers KS, Eaton M, Lerner NB. Children with single-ventricle physiology do not benefit from higher hemoglobin levels post cavopulmonary connection: results of a prospective, randomized, controlled trial of a restrictive versus liberal red-cell transfusion strategy. Pediatr Crit Care Med. 2011;12:39-45.

16. Kuo JA, Maher KO, Kirshbom PM, Mahle WT. Red blood cell transfusion for infants with single-ventricle physiology. Pediatr Cardiol. 2011;32: 461-8.

17. Willems A, Harrington K, Lacroix J, Biarent D, Joffe AR, Wensley D, et al. Comparison of two red-cell transfusion strategies after pediatric cardiac surgery: a subgroup analysis. Crit Care Med. 2010;38:649-56.
18. Guzzetta NA. Benefits and risks of red blood cell transfusion in pediatric patients undergoing cardiac surgery. Paediatr Anaesth. 2011;21:504-11.

19. Gauvin F, Lacroix J, Robillard P, Lapointe H, Hume H. Acute transfusion reactions in the pediatric intensive care unit. Transfusion. 2006;46:1899-908.

20. Sanchez R, Toy P. Transfusion related acute lung injury: a pediatric perspective. Pediatr Blood Cancer. 2005;45:248-55.

21. Bowman ME, Rebeyka IM, Ross DB, Quinonez LG, Forgie SE. Risk factors for surgical site infection after delayed sternal closure. Am J Infect Control. 2012;41: 464-5.

22. Kansy A, Jacobs JP, Pastuszko A, Mirkowicz-Malek M, Manowska M, Jezierska E, et al. Major infection after pediatric cardiac surgery: external validation of risk estimation model. Ann Thorac Surg. 2012;94:2091-5.

23. Grisaru-Soen G, Paret G, Yahav D, Boyko V, Lerner-Geva L. Nosocomial infections in pediatric cardiovascular surgery patients: a 4-year survey. Pediatr Crit Care Med. 2009;10:202-6.

24. Pasquali SK, Hall M, Li JS, Peterson ED, Jaggers J, Lodge AJ, et al. Safety of aprotinin in congenital heart operations: results from a large multicenter database. Ann Thorac Surg. 2010;90:14-21.

25. Jonas RA. Blood conservation guidelines for pediatric patients. Ann Thorac Surg. 2011;92:403-4.

26. Mou SS, Giroir BP, Molitor-Kirsch EA, Leonard SR, Nikaidoh H, Nizzi F, et al. Fresh whole blood versus reconstituted blood for pump priming in heart surgery in infants. N Engl J Med. 2004;351:1635-44.

27. Lacroix J, Demaret P, Tucci M. Red blood cell transfusion: decision making in pediatric intensive care units. Semin Perinatol. 2012;36:225-31.

28. Lacroix J, Hebert PC, Hutchison JS, Hume HA, Tucci M, Ducruet T, et al. Transfusion strategies for patients in pediatric intensive care units. N Engl J Med. 2007; $356: 1609-19$

29. Rouette J, Trottier H, Ducruet T, Beaunoyer M, Lacroix J, Tucci M, et al. Red blood cell transfusion threshold in postsurgical pediatric intensive care patients: a randomized clinical trial. Ann Surg. 2010;251:421-7.

30. Toledano B, Tucci M, Lacroix J. Red cell transfusion to cardiac patients: facts and fallacies. Pediatr Crit Care Med. 2011;12:107-8. 


\section{Ventilator and ICU Hours}

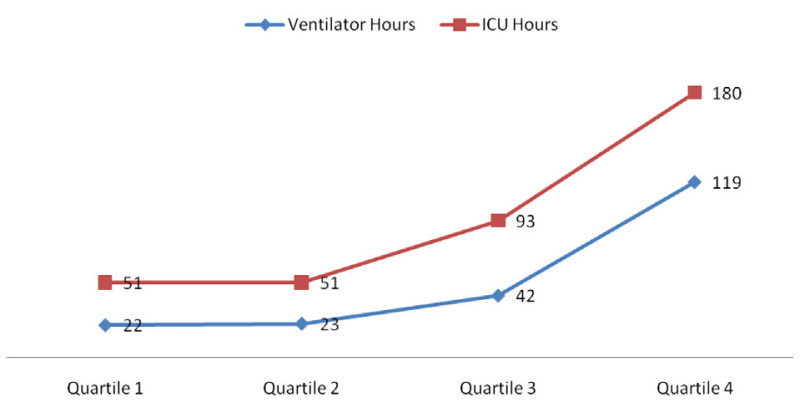

FIGURE E1. Median ventilator and intensive care unit $(I C U)$ hours stratified by quartile. 